\title{
Synthesis and Comparison of Three Novel $\beta$-Lactam Antibiotics using Diphenyl acetyl Chloride and 3,4,5 Trimethoxybenzoyl Chloride
}

\author{
Ariana Martinez, Arnauld Martinez \\ Western Center Academy 2345 Searl Pkwy, Hemet, CA 92543, U.S.A.; ariana145597@gmail.com
}

\begin{abstract}
Bacterial infections, often overlooked due to the publicity viral infections received in 2020, are resurging and becoming resistant to antibiotics at an alarming rate. Pharmaceutical companies are unwilling to invest in antibiotic research because the drug development process is no longer profitable. One explanation for the lack of success in drug development is that in the ten years required to ensure antibiotic safety, bacteria will already develop resistance to the new, experimental drug. The purpose of this project is to create three new $\beta$-lactam antibiotics and test their efficacy against both gram positive and gram negative bacteria. Additionally, a list of differences in the acyl chlorides, which may contribute to the antibiotics' differential success, will be compiled. These inferences will be explored and formally documented in a follow-up study to increase a company's chance of choosing an acyl chloride that creates a successful antibiotic. In this study we show how acyl chlorides with a smaller molar mass and fewer functional groups, and smaller surface area increase the efficacy of the antibiotic. Furthermore, we show how Diphenylacetyl Chloride demonstrates greater antibacterial effects than 3,4,5 Trimethoxybenzoyl Chloride to 6-APA and 7-AVCA as it has fewer functional groups and a smaller surface area. These findings influence the drug development process as they determine what factors make an effective antibiotic narrowing down the range of potentially effective acyl chlorides implemented in modern day antibiotics.
\end{abstract}

KEYWORDS: Antibiotic Development; Bacterial Resistance; Chemistry; Biology.

\section{- Introduction}

Bacterial infections were once considered incurable. Theologians and herbalists have long created solutions that allegedly cleanse the body of infections, but none had enough evidence to convince the scientific community of their efficacy. This changed in 1928 with the discovery of penicillin. Dr. Alexander Fleming became the first scientist to isolate and mass produce penicillin, the world's first bacteria fighting agent. ${ }^{1}$ to date, penicillin has saved over 200 million lives. Although Dr. Fleming was the first to introduce penicillin to the scientific community and prove its efficacy using the scientific method, an ancient Chinese transcript revealed laborers cultivated fungi containing penicillin-like compounds to treat bacterial infections two thousand years ago. ${ }^{2}$ Curiously, the vast majority of antibiotics synthesized today are based on natural molecules found in flowers, fungi, and animals. ${ }^{3}$ However, through the harmful combination of natural selection and an irresponsible distribution/consumption of drugs, bacteria have slowly developed resistance to many leading antibiotics. ${ }^{4,5}$ Infections that were once easily treatable with antibiotics have become incurable yet again.

As a result, there is an increased demand for new, more effective antibiotics that can treat those resistant bacterial. ${ }^{6}$ Despite this growing problem of antibiotic resistant bacteria, antibiotic production is no longer a profitable market, and the number of antibiotics approved by the FDA have decreased over $57 \%$ over the past 15 years. $^{7}$ This is because by the time antibiotics are developed for large scale distribution, the bacteria they are fighting have already become resistant to the antibiotic. ${ }^{7}$ With a tremendous number of combinations of acyl chlorides that have the potential to be effective antibiotics, there is simply no way to efficiently test all of the combinations. ${ }^{8}$ In response to this problem, our research aims to narrow down these combinations by determining what factors affect the effectiveness of an antibiotic.

In this work we developed three novel $\beta$-lactam antibiotics and tested their efficacy against gram positive and gram negative bacteria. Many antibiotics currently on the market have a small surface area making it easier for them to attach themselves on the infected cells. Hence, we aimed to minimize the surface area of our antibiotics in hopes of increasing the efficacy of the antibiotic. ${ }^{9}$ Therefore, we selected dipheneylacyl chloride and ${ }^{3,5}$, trimethoxybenzoyl chloride for our experiment as it minimizes the surface area the most while maintaining a reasonable price and synthesis procedure. If we chose to work with a smaller compound, we would not have had the materials to synthesize the antibiotic at the lab we were working in. This was the first time these antibiotics were synthesized in a lab. By comparing the structure of their functional groups and their surface areas to their efficacy, we were able to determine which factors create an effective antibiotic. 


\section{Methods}

Synthesis of stirred 6-APA solution:

Synthesis of stirred 6-APA solution to synthesize the first compound necessary for the analysis, under a fume hood, clamp $50 \mathrm{~mL}$ Erleenmeyer flask over a magnetic stirrer(previously cleaned with acetone). Continued by placing a magnetic stir bar into the flask along with $1.05 \mathrm{~g}$ sodium bicarbonate (CAS No. 144-55-8)d $09 \mathrm{~mL}$ deionized water. Left the mixture alone for 10-15 minutes, or until most sodium bicarbonate was dissolved. Proceeded by adding $3 \mathrm{~mL}$ of acetone, leaving the magnetic stir bar inside. Added 0.0025 moles of 6-APA (0.541 g 6-APA: CAS No. 551-16-6)), left until dissolved(took only minutes). Saved 3 drops of this solution in a labelled microcentrifuge for TLC comparison.

\section{Synthesis of Diphenylacetyl chloride solution:}

Synthesis of Diphenylacetyl chloride solution by securing a $15 \mathrm{~mL}$ conical tube with a clamp. Added 0.005 moles of Diphenylacetyl chloride(CAS No. 1871-76-7, M.W.230.69g/ $\mathrm{mol})$ and $1 \mathrm{~mL}$ of acetone. Capped tube and vortexed to dissolve mixture. In the scenario that the acyl chloride failed to dissolve, added another drop of acetone and vortex again. If the solution still failed to dissolve, continued adding one drop of acyl chloride until the solution is combined. Saved 3 drops of this Diphenylacetyl chloride(CAS No. 1871-76-7, M.W. $230.69 \mathrm{~g} / \mathrm{mol}$ ) solution in labelled microcentrifuge for TLC analysis.

\section{Analysis of reaction mixture using Thin Layer \\ Chromatography:}

Analysis of reaction mixture using Thin Layer Chromatography using a disposable pipette, transferred the acyl chloride solution dropwise every 5 minutes to the stirred solution of 6-APA until combined. This mixture was analyzed along with each component using TLC(thin layer chromatography). Labelled three TLC plates 5, 10, and 15. Each TLC plate must be marked with three lanes: one lane for 6-APA(labeled 6), one lane for reaction(labeled $\mathrm{R}$ ) and one lane food acyl chloride(A). Waited five minutes after the addition of the acyl chloride and then spotted the reaction solution in the middle lane of the TLC. Started elution. Spotted the reaction again after 10 minutes and 15 minutes. When the first two TLC plates (labelled 5 and 10 minutes) were complete, gently heated them to remove solvent, then stained with iodine to reveal the high RF product.

\section{Preparation of workup solutions:}

Preparation of workup solutions while the reaction was running, prepared workup solutions consisting of $10 \mathrm{~mL}$ deionized water in a $20 \mathrm{~mL}$ test tube, $2 \mathrm{~mL} 5 \mathrm{M}$ sulfuric acid in a $10 \mathrm{~mL}$ test tube, $6 \mathrm{~mL}$-butyl acetate(CAS No. 123-86-4) to a $10 \mathrm{~mL}$ test tube, while remembering to cool them in an ice bath.

\section{Liquid-Liquid separation of solution:}

Liquid-Liquid separation of solution in order to perform the Liquid-Liquid separation, took the previous solution of DiphenalAcetyl Chloride a(CAS No. 1871-76-7, M.W. $230.69 \mathrm{~g} / \mathrm{mol}) \mathrm{Nd} 6$-APA and performed the following steps. Using a clamp, held a $30 \mathrm{~mL}$ separatory funnel with the valve closed over a $50 \mathrm{~mL}$ beaker and poured the reaction mixture closed over a $50 \mathrm{~mL}$ beaker and poured the reaction mixture into the separatory funnel. Performed the first liquid-liquid extraction to remove most of the unreacted acyl chloride. To do this we added $6 \mathrm{~mL}$ n-butyl acetate(room temperature) to the solution and closed the separatory funnel. While holding the cap in place, inverted the separatory funnel and vented it by turning the valve $90^{\circ}$ and closed it (shook vigorously for a few seconds to mix the organic and aqueous solvents). Vented the separatory funnel and put the separatory funnel in a ring clamp (removed the cap). Drained the lower aqueous phase into the $50 \mathrm{~mL}$ beaker labelled AQ. Then drained the remaining n-butyl acetate solution into a $50 \mathrm{~mL}$ Erlenmeyer flask labelled BuAc extract and closed the valve. Then poured the aqueous phase AQ back into the separatory funnel. Performed the second liquid-liquid extraction to remove more of the unreacted acyl chloride by repeating step c. Saved the combined $\mathrm{BuAC}$ extracts and labelled them organic waste.

\section{Isolating antibiotic using vacuum filtrationy:}

Isolating antibiotic using vacuum filtration in preparation of the vacuum filtration took the AQ layer in the $50 \mathrm{~mL}$ beaker and added $6 \mathrm{~mL}$ of cold n-butyl acetate using a disposable pipet. Then added $5 \mathrm{M}$ sulfuric acid dropwise while stirring with a stir rod to dissipate the carbon dioxide gas. Additional drops led to less gas evolution, so started testing the $\mathrm{pH}$ of the lower aqueous solution using $\mathrm{pH}$ paper. Note that good stirring helped the derivative to move from the lower aqueous layer into the upper n-butyl acetate layer. When the sulfuric acid caused the solution to reach a $\mathrm{pH}$ of 2 carefully disposed of the remaining sulfuric acid solution. After this had been completed the solution was ready to undergo vacuum filtration. Secured a Büchner flask with a clamp and using a thick walled tubing, attached the flask's side arm to the vacuum trap. Carefully set a rubber bung on the head of the flask and put the Büchner funnel on top of the rubber bung with filter paper. Turned the vacuum on, and carefully put the suspension on the filter paper. After most of the moisture is extracted from the precipitate, carefully transferred the remaining solid into a glass vial. At this point we had isolated our antibiotic.

\section{Making dilutions of antibiotic:}

Making dilutions of antibiotic Antibiotics are considered most effective when they present great effect in small dilutions. Hence, we diluted our antibiotic to test if it was applicable in the antibiotic market today. First, we created stock solutions of 6-APA and 7-AVCA(7-AVCA: CAS No. 79349-82-9) derivatives $(10 \mathrm{mM})$. Next, we made a serial dilution of both derivatives (6-APA and 7-AVCA) with a dilution factor of $1 / 10$. Starting at $10 \mathrm{mM}$ and diluting to $1 \mu \mathrm{M}$. Left at least $500 \mu \mathrm{L}$ of each dilution.

\section{Testing purity using mass spectrometry:}

Testing purity using mass spectrometry using mass spectrometry we took a few drops of our 100 micro molar solution of our antibiotic. Entering it into the mass spectrometer, allowed particles to run through until results were clearly displayed on the computer. This step was extremely important, but it was very simple as the machine determines impurities in the solution due to their tiny size. [see discussion for graphs] 


\section{Testing efficacy against gram positive and gram negative} bacteria:

Testing efficacy against gram positive and gram negative bacteria in this experiment we tested against both gram positive and gram negative bacteria. First, obtained three agar test plates, one plate contained E. coli, and two contained Streptococcus: labelled each accordingly. Dipped a sterile swab into the inoculum tube of bacteria and rotated the swab against the side of the tube (above fluid level) using firm pressure, to remove excess fluid. Inoculated the surface of the agar plate by streaking the swab over the entire surface of the plate. Rotated $90^{\circ}$ and repeated again. (4x). Rimmed the plate with the swab to pick up any excess liquid and then allowed the plate to sit at room temperature for 3-5 minutes, but no more than 15 minutes. Wiped down a pair of forceps with alcohol swabs. Use the forceps to dip a circular filter paper in each diluted solution of 6-APA and 7-AVCA derivatives (see step 13). Pulled the filter paper out, shook off any excess solution, then placed it on the agar plate appropriately using template I. Once all the disks were in place, covered and inverted the plates and labelled each position on the plate. Incubated the disks for three days (35 ${ }^{\circ} \mathrm{C} \pm 2{ }^{\circ} \mathrm{C}$ with no exposure to $\mathrm{CO}_{2}$ ). Following incubation, measured each disk's zone size to the nearest millimeter using a ruler. Then measured from the center of the disk to a single point on the circumference of the zone where a visible edge is present.

\section{- Results}

From the UV Thin Layer Chromatography (Figure 3), we can determine the relative purity of the antibiotics synthesized in our experiment. The long streaks and relative absence of separated stains reveals 3,4,5 Trimethoxybenzoyl Chloride bonded to 6-APA was over $90 \%$ pure, Diphenylacetyl Chloride bonded to 6-APA was over $80 \%$ pure, and 3,4,5 Trimethoxybenzoyl Chloride bonded to 7 -AVCA was over $50 \%$ pure. Additionally, data from the mass spectrometry graphs reveal the chemical makeup of our antibiotic (Figure 4). Since the mass spectrometer was run in positive ion mode, we expect to see positive ions bonded to our antibiotic $(\mathrm{Na}+, \mathrm{K}+, \mathrm{H}+)$. The presence of some unidentified spikes and unreacted reagents suggests the presence of impurities, responsible for the low purity of 3,4,5 Trimethoxybenzoyl Chloride bonded to 7-AVCA.

The larger the zone of clearance $(\mathrm{ZoC})$ the more efficient our antibiotic was as we aim to inhibit the largest number of bacteria at a small dilution (Figures 1 and 2). The zones of clearance on the agar plates reveals Diphenylacetyl chloride bonded to 6-APA is the most effective antibacterial agent, inhibiting bacterial growth from concentrations of $10 \mathrm{mM}$ to $100 \mu \mathrm{M}$. 3,4,5 Trimethoxybenzoyl chloride bonded to 7-AVCA exhibited antibacterial effects in its highest concentration: $10 \mathrm{mM}$. 3,4,5 Trimethoxybenzoyl chloride bonded to 6-APA, however, displayed no antibacterial effects. Only two strains of bacteria were tested, so further testing would be required to dismiss or strengthen my antibiotics' potential for antibacterial activity.

Some notable differences are Diphenylacetyl chloride, exhibiting antibacterial effects to the largest degree, has a smaller surface area than 3,4,5 Trimethoxybenzoyl chloride. The functional groups surrounding the benzene ring on 3,4,5 Tri methoxybenzoyl chloride are electrophilic, while the benzene rings on Diphenylacetyl chloride are relatively inert. Additionally, Diphenylacetyl chloride exhibits two benzene rings indirectly bonded to its acyl group, while 3,4,5 Trimethoxybenzoyl chloride exhibits one benzene ring directly bonded to its acyl groups.
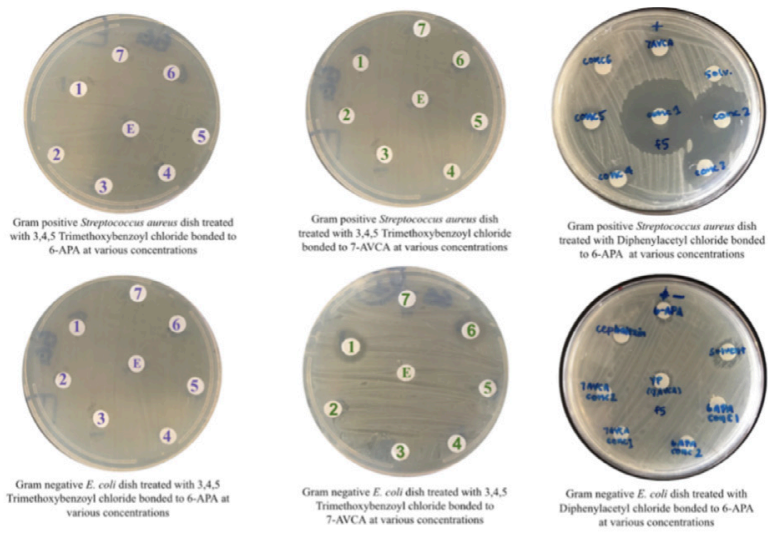

Figure 1: Gram Positive and Gram Negative plates treated with developed antibiotics.
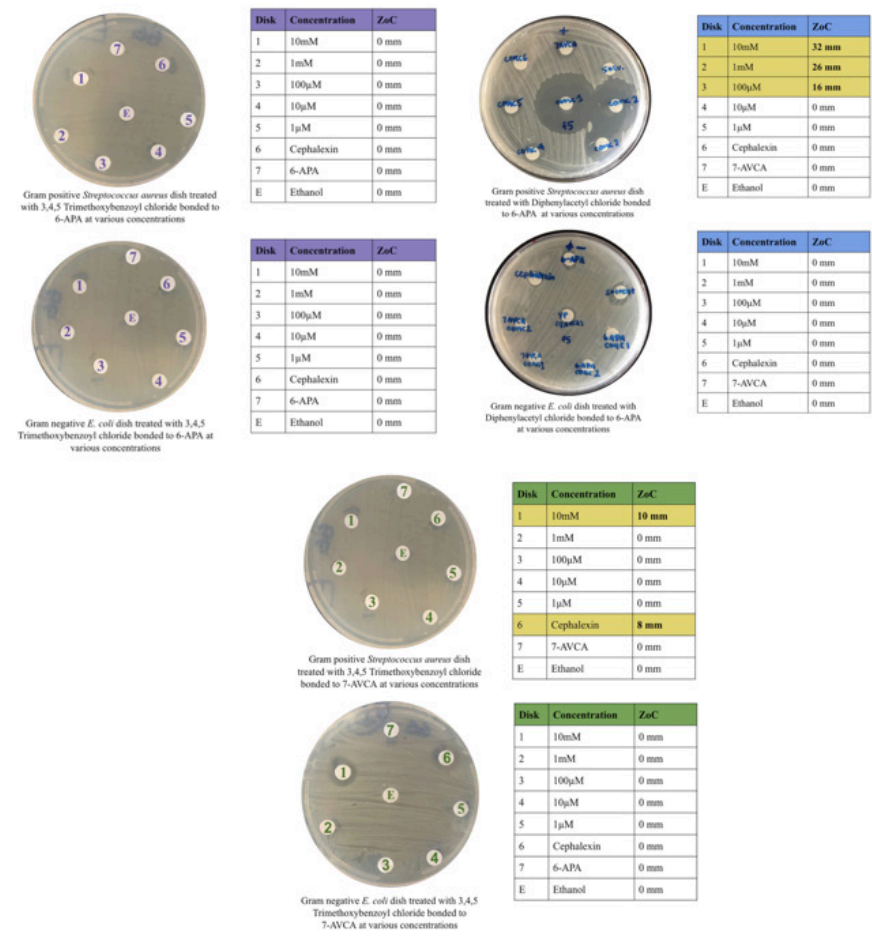

Figure 2: Closer look at each dish with table describing zone of clearance.
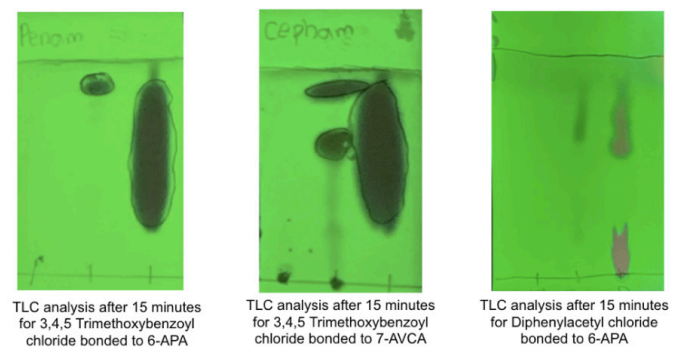

Figure 3: Thin Layer Chomatography (TLC) plates. 
In the TLC analysis reactants are on the far left lane, coproducts are located on the middle lane, and products are located on the far right lane.\}

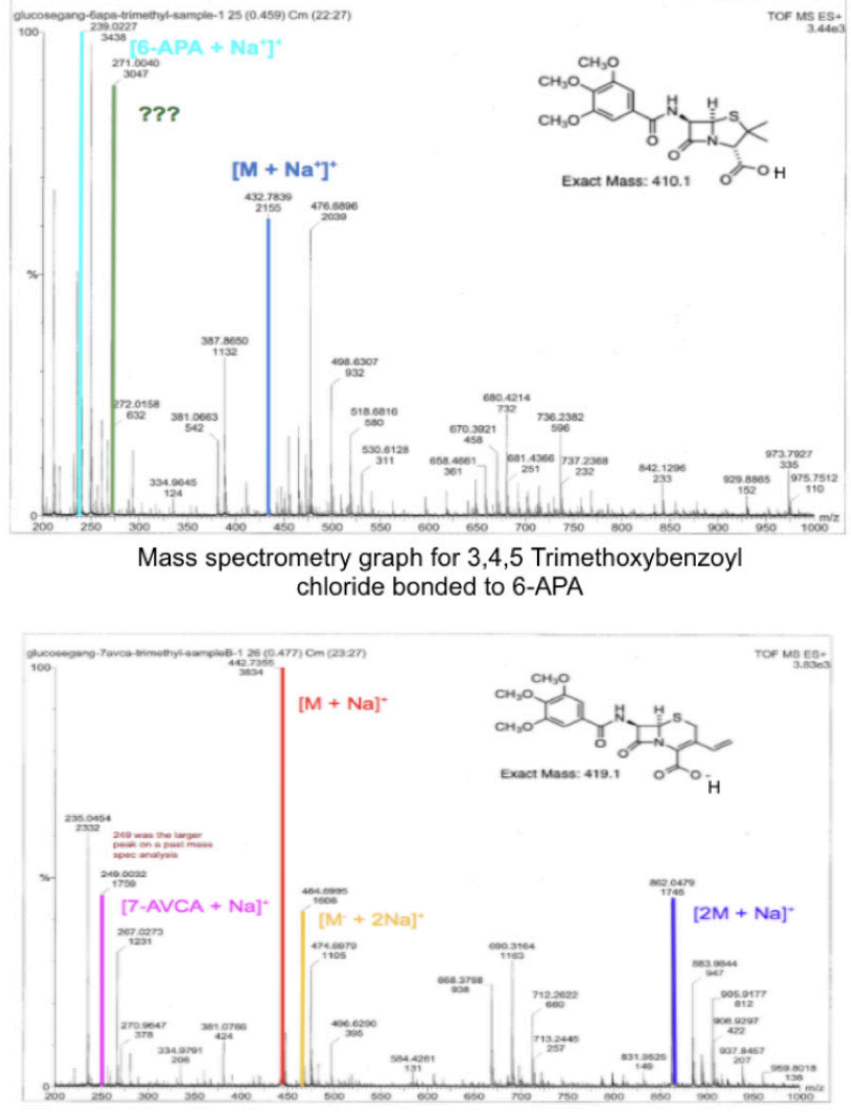

Mass spectrometry graph for 3,4,5 Trimethoxybenzoyl chloride bonded to 7-AVCA

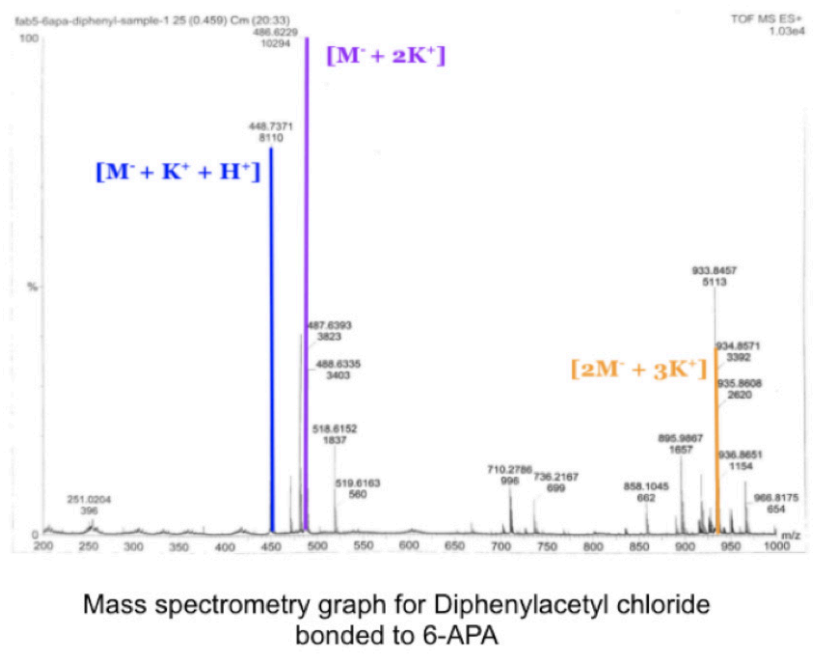

Figure 4: Mass Spectrometry.

\section{- Discussion}

Although further testing would be required to fully dismiss or strengthen our antibiotics' potential for antibacterial activity, the success of the acyl chlorides in creating a useful antibiotic behaves in a predictable manner. Diphenylacetyl chloride has the smallest surface area and exhibits antibacterial effects to the greatest degree. This agrees with data from antibiotics currently on the market: antibiotics whose acyl chloride has a smaller surface area is more effective at inhibiting bacterial growth. Thienamycin, for example, is a B-lactam antibiotic utilizing one of the smallest acyl chlorides known to man. Yet, Thienamycin is the most potent naturally produced antibiotic. It is so potent, pure Thienamycin is not practical for clinical use. Stable derivatives have been made for medicinal consumption.

Additionally, the methoxy functional groups (-OCH3) surrounding 3,4,5 Trimethoxybenzoyl Chloride act as an electrophile. However, the benzene rings in Diphenylacetyl chloride are relatively inert. The success of antibiotics like Diphenylacetyl chloride bonded to 6-APA and Penicillin G suggest a negative correlation between electrophilic functional groups and antibacterial efficacy. In fact, Amoxicillin, containing the nucleophilic hydroxyl group, extends this hypothesis to encompass all functional groups: electrophilic groups decrease the efficacy of B-Lactam antibiotics while nucleophilic groups increase the efficacy of B-Lactam antibiotics.

Finally, although not tested in our experiment, we predict a smaller molar mass increases the efficacy of antibiotics. Thienamycin, Penicillin G, and Amoxicillin are among the most effective antibiotics available today, they weigh $273 \mathrm{amu}, 334$ amu, and $365 \mathrm{amu}$ respectively, compared to our antibiotics weighing over $410 \mathrm{amu}$. These three characteristics, or subset thereof, are found in the vast majority of useful antibiotics. As a result, choosing an acyl chloride for a new antibiotic does not need to be random. Certain acyl chlorides are more promising at creating much-needed antibiotics than others.

\section{Acknowledgements}

I acknowledge and thank Dr. Jenifer Nalbandian from California Baptist University for donating her time to oversee all the lab work associated with this experiment.

\section{References}

1. "Alexander Fleming Discovery and Development of Penicillin - Landmark." American Chemical Society, www.acs.org/content/acs/en/education/. whatischemistry/landmarks/flemingpenicillin.html.

2. "Chinese Flower Has Evolved to Be Less Visible to Pickers." The Guardian, Guardian News and Media, 20Nov.2020,

www.theguardian.com/environment/2020/nov/ 20/chinese-flower-fritillaria-delavayi-evolved-lessvisible-pickers.

3. Cowan, M M. "Plant Products as Antimicrobial Agents." Clinical Microbiology Reviews, American Society for Microbiology, Oct. 1999, www.ncbi.nlm.nih.gov/pmc/articles/PMC88925/. .

4. Role of Microbes in Ecosystems, globalchange.umich.edu/globalchange1/current/ lectures/kling/microbes/microbes.html.

5. "What Is Antibiotic Resistance.

"What Is Antibiotic Resistance | Antibiotic

Resistance| Health \& Senior Services, health.mo.gov/safety/antibioticresistance/generalinfo.php.

6. "Current Report." Centers for Disease Control and Prevention, Centers for Disease Control and Prevention, 30 Nov. 2020, www.cdc.gov/antibiotic-use/ 
stewardship-report/current.html.

7. Lowe, Derek. "The Latest on Drug Failure and Approval Rates." In the Pipeline, 9 May 2019, blogs.sciencemag.org/pipeline/archives/2019/05/09/ the-latest-on-drug-failure-and-approval-rates.

8. Synergistic Antibiotic Combinations: Topics by Science.gov, www.science.gov/topicpages/s/ synergistic+antibiotic+combinations.html.

9. Ojkic, Nikola, D. Serbanescu, and S. Banerjee. "Surface-to-Volume Scaling and Aspect Ratio P reservation in Rod-Shaped Bacteria." ELife, ELife Sciences Publications, Ltd, 28 Aug. 2019, elifesciences.org/articles/47033.

\section{Author}

Ariana is a Sophomore attending Western Center Academy in Hemet California. Her passion in both chemistry and biology led to her interest in antibiotic development. In the future Ariana hopes to pursue a career in internal medicine. Outside of school, Ariana is a varsity swimmer and avid skier. 